\title{
Effect of neutral winds on the creation of non-specular meteor trail echoes
}

\author{
Freddy Galindo $^{1}$, Julio Urbina ${ }^{1}$, and Lars Dyrud ${ }^{2}$ \\ ${ }^{1}$ Communications and Space Sciences Laboratory, Department of Electrical Engineering, The Pennsylvania State University, \\ University Park, PA, USA \\ ${ }^{2}$ OmniEarth, Inc. 2015; 251 18th Street South - Suite 650, Arlington, VA, USA
}

Correspondence: Julio Urbina (jvu1@ psu.edu)

Received: 25 June 2020 - Discussion started: 14 July 2020

Revised: 4 June 2021 - Accepted: 8 June 2021 - Published: 23 July 2021

\begin{abstract}
Non-specular meteor trail echoes are radar reflections from plasma instabilities that are caused by fieldaligned irregularities. Meteor simulations are examined to show that these plasma instabilities, and thus the associated meteor trail echo, strongly depend on the meteoroid properties and the characteristics of the atmosphere in which the meteoroid is embedded. The effects of neutral winds, as a function of altitude, are analyzed to understand how their amplitude variability impacts the temporal-space signatures of non-specular meteor trail echoes present in very high-frequency (VHF) radar observations. It is found that amplitudes of the total horizontal neutral wind smaller than $0.6 \mathrm{~m} \mathrm{~s}^{-1}$ do not provide the right physical conditions to enable the genesis of non-specular meteor echoes. It is also found that a $0.0316 \mu \mathrm{g}$ meteoroid traveling at $35 \mathrm{~km} \mathrm{~s}^{-1}$ can be seen as a meteor trail echo if the amplitudes of horizontal neutral winds are stronger than $15 \mathrm{~m} \mathrm{~s}^{-1}$. In contrast, a $0.316 \mu \mathrm{g}$ meteoroid, traveling at the same speed, requires horizontal winds stronger than $1 \mathrm{~m} \mathrm{~s}^{-1}$ to be visible as a meteor trail echo. The neutral velocity threshold illustrates how simulations show that no trail echo is created below a critical wind value. This critical wind value is not mapped directly to radar observations, but it is used to shed light on the physics of meteor trails and improve their modeling. The meteor simulations also indicate that time delays on the order of hundreds of milliseconds or longer, between head echoes and non-specular echoes, which are present in VHF backscatter radar maps, can be a consequence of very dense plasma trails being affected by weak horizontal neutral winds that are smaller than $1 \mathrm{~m} \mathrm{~s}^{-1}$.
\end{abstract}

\section{Introduction}

Day and night, meteoroids smaller than a grain of sand penetrate the Earth's upper atmosphere and generate meteor plasma trails at altitudes between 70 and $140 \mathrm{~km}$. Meteor research is typically conducted with photographic and TV cameras and specular meteor radars. Specular radars detect echoes from the trail of ionization formed perpendicularly to the radar $\boldsymbol{k}$ vector by a meteoroid during atmospheric entry. This specular condition requires that only trails formed perpendicularly to the radar $\boldsymbol{k}$ vector reflect strongly without destructive interference (Ceplecha et al., 1998).

For more than 2 decades, scientists have studied two new types of radar meteor reflections. These reflections are known as meteor head echoes and non-specular trails, typically observed with high-power and large-aperture (HPLA) radars. Non-specular meteor echoes are radar reflections from meteor plasma instabilities that are generated from field-aligned irregularities (FAIs); i.e., non-specular meteor echoes are detected when pointed perpendicularly to the Earth's magnetic field $\boldsymbol{B}$ (Chapin and Kudeki, 1994; Zhou et al., 2001; Oppenheim et al., 2001, 2003, 2014; Dyrud et al., 2002; Chau et al., 2014; Oppenheim and Dimant, 2015; Dimant and Oppenheim, 2017a, b; Sugar et al., 2018, 2019). Although substantial progress has been made in meteor trail studies, we still do not understand the degree to which meteoroid and atmospheric properties affect the creation of non-specular meteor trail echoes. Specifically, this paper investigates the effect of horizontal atmospheric winds on the development and progression of non-specular meteor trails. Our goal is to understand how a realistic vertical wind profile will influence echo structures routinely seen in VHF range-time-intensity 
maps. Assessing the effects of neutral wind profiles on a nonspecular meteor trail is essential for scientific studies of meteors, the Earth's upper atmosphere, and engineering applications of meteors (Dyrud et al., 2005, 2007; Malhotra et al., 2007). To determine the effect of a neutral wind profile on non-specular trail progression, we augmented the physical model of the evolution of an individual meteor from direct atmospheric entry to trail instability and diffusion (Dyrud et al., 2005, 2007) by including the most recent empirical climatological models with the corresponding ionospheric and atmospheric parameters.

The work reported here and the model we have constructed seek to understand how a meteoroid of a given mass, velocity, and entry angle will produce different non-specular radar signatures due to different values of horizontal neutral winds. To characterize these effects, we present artificial radar rangetime-intensity (RTI) images over Salinas, Puerto Rico, with different meteor properties such as mass and velocity. These simulations are further compared with actual radar observations of non-specular meteor trail echoes collected over Salinas, Puerto Rico, during the Coqui-II NASA Rocket Campaign (Urbina et al., 2000). This work complements the research described in Hinrichs et al. (2009) and Dyrud et al. (2011) and effectively demonstrates that non-specular meteor trail echoes can exhibit unique radar signatures due to the variability of horizontal neutral winds. The implications of our simulations are extended to interpret meteor trail data collected with the $50 \mathrm{MHz}$ Jicamarca radar (Chau and Galindo, 2008). Unlike the radar used during the Coqui-II campaign, Jicamarca radar usually, but not always, observes both meteor head echoes and their associated non-specular meteor trail echoes. These two types of meteor echoes provide a unique opportunity to correlate changes in the meteor head echo with variations in the non-specular echo and therefore help to determine whether the radar signature in the trail echo is generated by the parent meteoroid or the background atmosphere.

This paper is organized as follows. In Sect. 2, we describe the new features of the non-specular meteor trail echo simulator. In Sect. 3, we analyze the effect of horizontal neutral wind on the progression of meteor trails, in particular, the importance of horizontal neutral winds sustaining plasma instabilities in the meteor trail. Section 4 presents and discusses radar observations of meteor trail echoes that resemble meteor simulations that capture the effect of neutral winds, while Sect. 5 summarizes the conclusions of our studies.

\section{Non-specular meteor trail echo simulator}

The non-specular meteor trail echo simulator (NSMES) is fully described in Dyrud et al. $(2005,2007)$ but has been updated to include the most recent climatological models for the atmospheric and ionospheric drivers and background conditions that we report in this paper. As explained in these papers, the model starts by computing the amount of ablated particles created behind the meteoroid body. These energetic particles are then used to calculate the amount of ionization created in the trail. Here we assume that the ionization created in the trail is initially distributed in a cylindrical volume defined by the initial radius. At this point, the trail is expanded by either ambipolar diffusion or turbulent diffusion to simulate the absence or presence of plasma instabilities in the trail during its evolution (Dyrud et al., 2001; Yee and Close, 2013). The plasma instability analysis is based on meteor Farley-Buneman gradient-drift (FBGD) instability reported in Dyrud et al. (2002) and Oppenheim et al. (2001, 2003). NSMES assumes that a non-specular meteor trail echo is created because the trail becomes Bragg reflective at altitudes where plasma instabilities can develop (Dyrud et al., 2002). The results of the simulations produce artificial radar RTI images that we use to compare with Coqui-II and Jicamarca meteor observations.

The atmospheric/ionospheric parameters needed to execute NSMES include electron density, atmospheric neutral mass density, neutral temperature, and neutral winds for a specific location and time. These numerical values of the properties of the atmosphere are obtained from the latest versions of these climatological models: the NRLMSISE-00 empirical model of the atmosphere (Picone et al., 2002), Horizontal Wind Model HWM14 (Drob et al., 2008, 2015), and the International Reference Ionosphere IRI-2016 (Bilitza and Reinisch, 2008; Bilitza, 2018). Our model and the associated software can be executed in a general-purpose PC-based system. It can easily be adapted and combined with other tools to study very large meteor populations. In contrast, as far as we know, more sophisticated 3D meteor models require supercomputer clusters and do not fully simulate the actual extent of a meteor trail or produce results that can be closely compared to 2D observational data. Although our numerical model is a simplified representation of the meteor physics, it can produce very good and fine details such as those reported in this paper. Our model can be used to account for and understand the statistical outcome of thousands of meteors acting collectively on the Earth's upper atmosphere.

Figure 1 displays an example of an artificial RTI, produced using NSMES, showing a simulated head echo and the non-specular meteor trail. In this plot, meteor line density in units of electron per micron for the thin head echo is shown in the vertical color bar. The non-specular trail color indicates the FBGD instability growth rate in units of inverse seconds. The meteor generating this head echo and non-specular trail had a known mass of $1 \mu \mathrm{g}$, traveling at $30 \mathrm{~km} \mathrm{~s}^{-1}$, at a zenith angle of $45^{\circ}$. Vertical profiles of the corresponding atmosphere/ionosphere parameters covering the same altitude range for the simulations are presented in Fig. 2. These climatological properties were chosen to characterize the atmosphere and ionosphere over Salinas, Puerto Rico, at 00:00 UT on 1 January 1998. Of these three vertical profiles, the horizontal neutral wind vertical profile is the 
only atmospheric parameter used by NSMES that exhibits more amplitude variability in altitude. This characteristic of neutral winds influences the structure of the resulting meteor trail echo if for instance neutral wind values drastically change in a region that is smaller than or comparable in size to the meteor trail. These results are further discussed and presented in the following section.

\section{Effect of horizontal neutral winds on the space-time evolution of non-specular trails}

We deployed a similar simulation approach described in Hinrichs et al. (2009) and Dyrud et al. (2011) but focus specifically on the impact of a more realistic vertical profile of horizontal neutral wind on the space-time meteor trail evolution at a specific mid-latitude location: Salinas, Puerto Rico. We kept the main elements and considerations of their simulation procedures but focused on two specific case studies to understand how the amplitude variability of neutral wind values affects the duration of non-specular meteor trail echoes.

Since we are interested in comparing trail durations between simulations and observations, we computed simulated trail duration at a given altitude using this simple criterion: subtracting time values between the longest duration of nonspecular echo and the corresponding onset of head echo/nonspecular echo in an artificial RTI image, as illustrated in Fig. 1. For example, for this figure, the trail duration is about $7.5 \mathrm{~s}$, at an altitude of $97.5 \mathrm{~km}$. The corresponding range span of the non-specular echo is approximately $10 \mathrm{~km}$.

To assess the impact of horizontal neutral winds on the time duration of non-specular meteor trail echoes, simulations were produced using horizontal neutral winds between 2.5 and $100 \mathrm{~m} \mathrm{~s}^{-1}$, meteoroid masses between $3.16 \times 10^{-9}$ and $3.16 \times 10^{-5} \mathrm{~g}$, and speeds between 11 and $72 \mathrm{~km} \mathrm{~s}^{-1}$. We used these meteoroid parameters because they represent a commonly detected class of meteoroid (Mathews et al., 2001; Dyrud and Janches, 2008). Additionally, NSMES assumes that meteoroids have a chondritic composition (45\% oxygen, $15 \%$ iron, $9 \%$ magnesium, and $31 \%$ silicon) with a mean atomic mass weighing about $30 \mathrm{amu}$ and have an entry zenith angle of $45^{\circ}$. Our analysis also employed the atmospheric properties shown in Fig. 2a and c. These two figures display typical vertical amplitudes of neutral mass and electron densities used in the simulations reported in this paper, which were used for a case study of meteoroids traveling with a known velocity of $35 \mathrm{~km} \mathrm{~s}^{-1}$. Figure $2 \mathrm{~b}$ represents the vertical amplitude variability of neutral winds. This specific vertical profile was used to produce meteor simulations shown in Fig. 1.

The outcomes of the case study are displayed in Fig. 3a and $b$. Several general conclusions can be inferred upon further examination of these two figures. For example, from Fig. 3a, it is clear that meteoroids with small masses require stronger neutral winds to create non-specular echoes. This means that meteor trails with low electron densities need stronger winds to keep the total electron drifts large enough to produce and sustain plasma instabilities and therefore enable the genesis of non-specular meteor trail echoes. Additional details about the meteor physics can be found in Oppenheim et al. (2001, 2003) and Dyrud et al. (2002). Further inspection of Fig. 3a shows that a $0.01 \mu \mathrm{g}$ meteoroid can create non-specular echoes if winds are stronger than $60 \mathrm{~m} \mathrm{~s}^{-1}$, while a $0.0316 \mu \mathrm{g}$ meteoroid requires winds stronger than $15 \mathrm{~m} \mathrm{~s}^{-1}$ to give rise to non-specular echoes. It can also be seen from Fig. 3a that if these meteoroids travel at $35 \mathrm{~km} \mathrm{~s}^{-1}$ and have masses smaller than $0.01 \mu \mathrm{g}$, they will never produce non-specular echoes. We repeated extensive simulations of meteoroids traveling with other velocity values. Still, we kept the same range of mass values and neutral winds to determine whether other trends could emerge by changing velocity values. However, the results of these simulations produced similar patterns to those depicted in Fig. 3a. For instance, if a meteoroid travels at $15 \mathrm{~km} \mathrm{~s}^{-1}$, masses larger than $1 \mu \mathrm{g}$ are required to create non-specular meteor trail echoes for the same range (between 2.5 and $100 \mathrm{~m} \mathrm{~s}^{-1}$ ) of horizontal neutral winds. In comparison, a meteoroid that travels at $55 \mathrm{~km} \mathrm{~s}^{-1}$ needs masses greater that $0.1 \mu \mathrm{g}$ to produce nonspecular meteor trail echoes with similar neutral wind ranges.

Another important implication of our meteor simulations is that trail duration linearly increases as a function of the amplitude of horizontal neutral winds to power 0.4 for the corresponding range of meteoroid masses mentioned earlier. This relationship became apparent when plotting trail duration as a function of neutral winds and produced a squareroot signature. The exponent 0.4 was determined by choosing a value that minimized the root-mean-square error of the curves. Three examples of simulated non-specular trail duration as a function of horizontal neutral winds to the power 0.4 that corresponds to meteoroid masses of $3.16,1$, and $0.31 \mu \mathrm{g}$ are shown in Fig. 3b. This empirical relationship between trail duration and neutral wind amplitudes provides insight to formulate practical and straightforward equations that could easily give an approach to determine meteor trail duration when considering different mass scenarios. This outcome is complementary to other relationships between non-specular echoes and the meteoroid mass and speed that are provided in Dyrud et al. (2011).

Perhaps the most important result of these simulations can be expressed with this probing question: is there a minimum value of horizontal neutral wind to enable non-specular meteor trail echoes? We think the answer is "yes", at least for the range of values of meteoroid masses and speeds presented in this paper. We conducted extensive simulations with similar meteoroid parameters and climatological atmospheric conditions to those that produced the results exhibited in Fig. 3a but restricted neutral wind values between 0.2 and $10 \mathrm{~m} \mathrm{~s}^{-1}$. Some of the outcomes of these simulations are shown in Fig. 4. In this example, the meteor trail is traveling at $35 \mathrm{~km} \mathrm{~s}^{-1}$ with masses between $3.16 \times 10^{-9}$ 


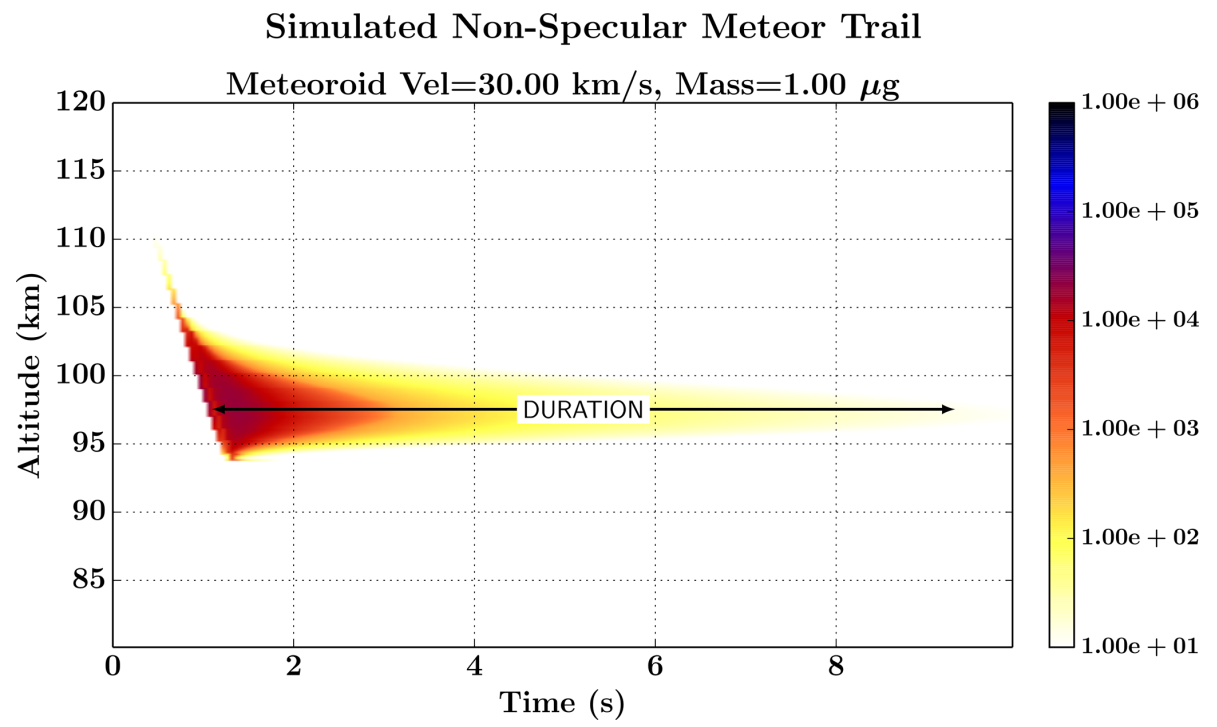

Figure 1. Simulated meteor head echo and non-specular meteor trail echo. This simulation was obtained using a $1 \mu \mathrm{g}$ meteoroid, traveling at $30 \mathrm{~km} \mathrm{~s}^{-1}$ at a $45^{\circ}$ entry angle. The duration of the non-specular echo is approximately $8 \mathrm{~s}$. The color in the non-specular meteor trail echo indicates the plasma instability growth rate in $\mathrm{s}^{-1}$, while for the simulated head echo it is the electron line density per meter divided by $10^{6}$ (units chosen such that they appear on the same scale).

(a)

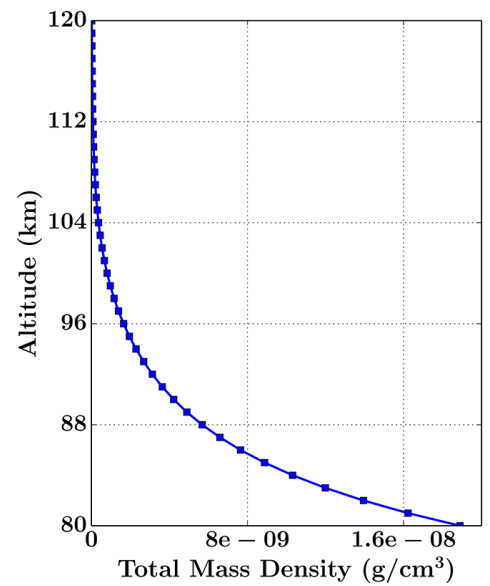

(b)

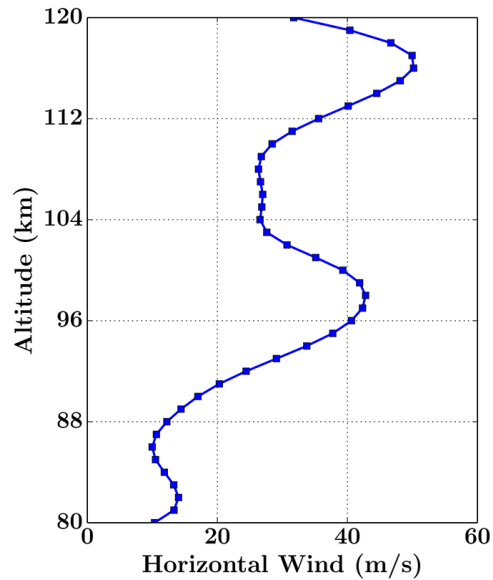

(c)

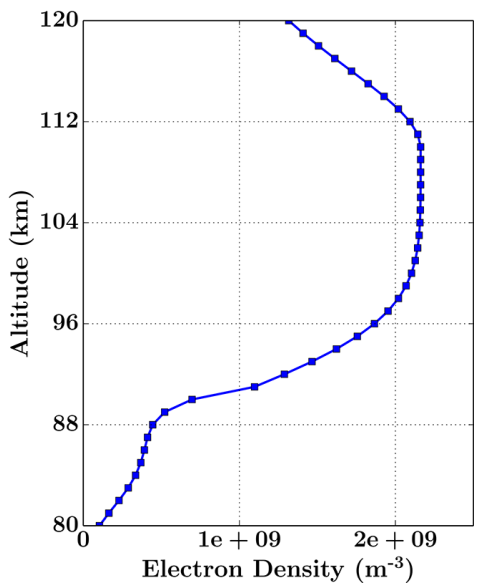

Figure 2. Corresponding atmospheric properties used to simulate the non-specular meteor echo shown in Fig. 1. (a) Background neutral density, (b) horizontal wind speeds, and (c) background electron density.

and $3.16 \times 10^{-5} \mathrm{~g}$. It can be seen that horizontal neutral wind values smaller than $0.6 \mathrm{~m} \mathrm{~s}^{-1}$ do not favor the development of non-specular echoes. Some of the implications of these results are further discussed in the next section.

\section{Discussion}

The first important implication of our analysis is captured in Fig. 5a. This artificial RTI shows a simulated head echo and non-specular meteor trail with the same physical characteristics of the parent meteoroid as those that were used to produce Fig. 1. The corresponding vertical profiles of the atmosphere/ionosphere parameters covering the same altitude range of these simulations are similar to those in Fig. 2a and $\mathrm{c}$, but the corresponding vertical profile of the horizontal neutral wind is displayed in Fig. 5b. These climatological properties were chosen to characterize the atmosphere and ionosphere over Salinas, Puerto Rico, at 00:00 UT on 2 February 1998. Basically, we determined that multiple non-specular echoes can be created from the same parent meteoroid mass as it makes its journey through a verticalhorizontal wind profile that contains very sharp wind gradients such as those depicted in Fig. 5b. The absence of non- 
(a)

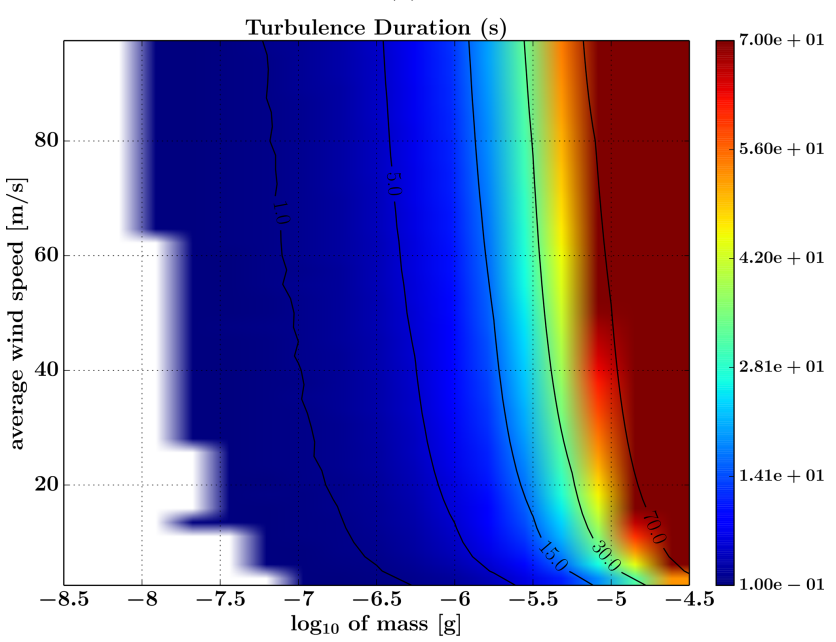

(b)

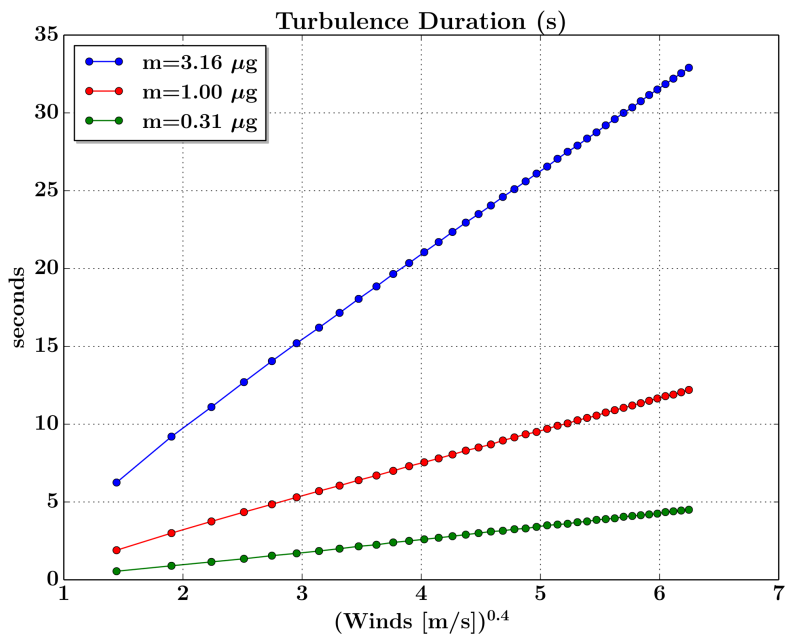

Figure 3. (a) Meteor trail simulations to investigate the influence of neutral winds on trail duration. These simulations used a meteoroid speed of $35 \mathrm{~km} \mathrm{~s}^{-1}$ and masses between $3.16 \times 10^{-9}$ and $3.16 \times$ $10^{-5} \mathrm{~g}$ and neutral wind amplitudes between 2.5 and $100 \mathrm{~ms}^{-1}$. (b) Trail duration versus winds for three masses. Notice the linear relationship between duration and winds with the power of 0.4 .

specular meteor trail echoes near $96 \mathrm{~km}$ altitude in the RTI of Fig. 5a is compatible with very low wind values that resemble wind shear effects near $96 \mathrm{~km}$, as shown in Fig. $5 \mathrm{~b}$. Notice that neutral wind values are very small near $96 \mathrm{~km}$, but their magnitude rapidly increases below or above this altitude to sustain plasma instabilities. Our result suggests that careful consideration of neutral wind effects on non-specular meteor trail evolution must be considered when interpreting meteor radar reflections. Ignoring neutral wind effects could lead to misinterpretation of these echo signatures and associated them, for example, with antenna beam side lobes or physical fragmentation of meteoroids.

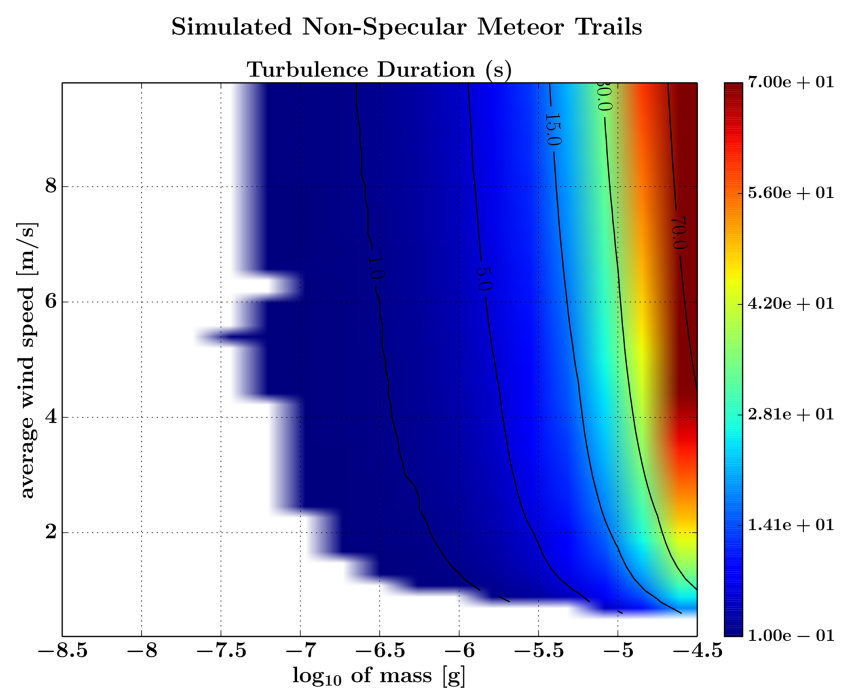

Figure 4. Minimum neutral winds vs. meteoroid mass. Notice that neutral winds smaller than $0.6 \mathrm{~m} \mathrm{~s}^{-1}$ do not create non-specular meteor trail echoes (i.e. plasma instabilities cannot be sustained in the trail).

The second implication of our studies is related to the time delay between head echo and non-specular meteor trail. One example showing this effect is presented in Fig. 6. This RTI plot shows a head echo and non-specular meteor trail echo that were simulated using a $5 \mu \mathrm{g}$ meteoroid traveling at $20 \mathrm{~km} \mathrm{~s}^{-1}$. The corresponding atmospheric properties used in this simulation are shown in Fig. 2a and c. Still, the amplitude of the vertical profile of the neutral wind is maintained constant and equal to $2.2 \mathrm{~m} \mathrm{~s}^{-1}$. We chose this low value of neutral wind to demonstrate that it is possible for non-specular meteor trail echo to take at least $500 \mathrm{~ms}$ to be created after the meteoroid has traveled through such low, neutral wind values. This effect is clearly illustrated in Fig. 6. It indicates that studies of non-specular meteor echoes as a function of atmospheric properties are critical when interpreting observational meteor reflections obtained with radar sensors.

We have just described two scenarios that can manifest themselves when neutral wind amplitudes are close to a critical value that is needed to sustain meteor plasma instabilities, which in turn will create non-specular meteor reflections. These results indicate that the impact of neutral winds on trail evolution is a plausible and complementary explanation of the characteristics exhibited by VHF radar reflections such as those reported in Close et al. (2004), Malhotra et al. (2007), Dyrud et al. (2007), Malhotra and Mathews (2009), and Sugar et al. (2010).

Further, our simulations here are compared with nonspecular meteor echoes collected during the Coqui-II campaign (Urbina et al., 2000). We extend the outcomes of our simulations to analyze head echoes and non-specular meteor echoes detected with the Jicamarca VHF radar during the $\eta$ Aquarids campaign (Chau and Galindo, 2008). 
(a) Simulated Non-Specular Meteor Trail

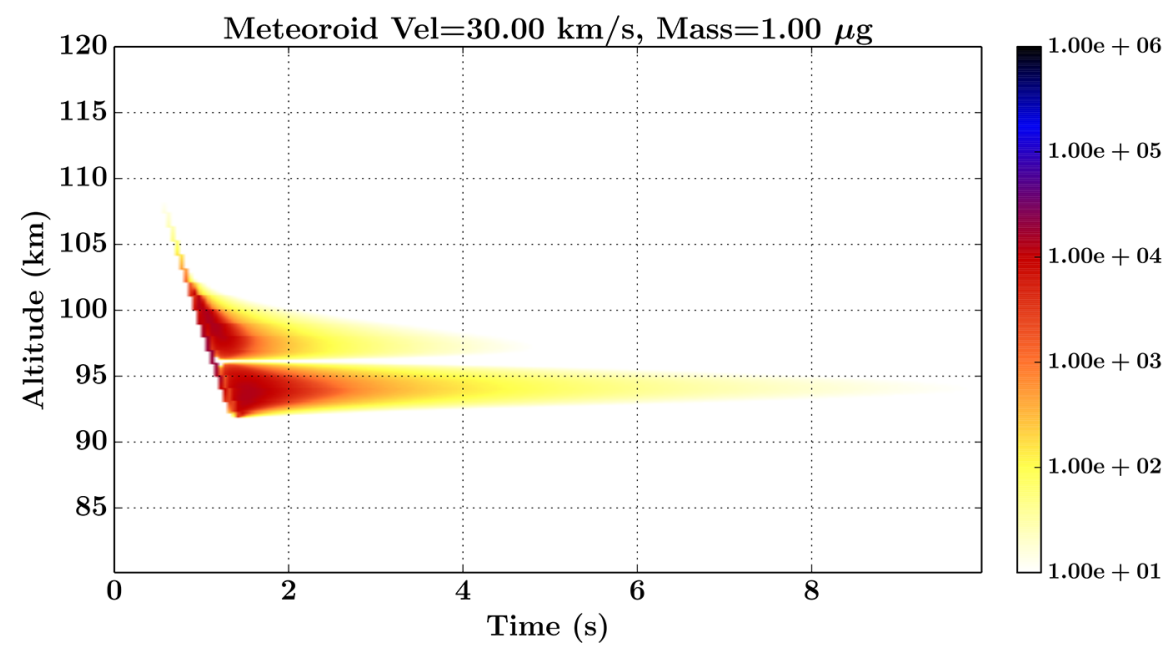

(b)

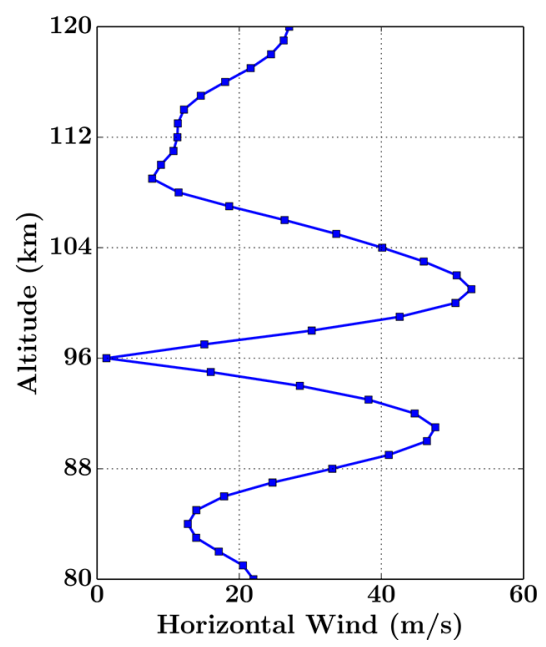

Figure 5. (a) Simulated non-specular meteor trail using a $1 \mu \mathrm{g}$ meteoroid traveling at $30 \mathrm{~km} \mathrm{~s}^{-1}$. (b) Corresponding neutral wind vertical profile. Notice strong wind shears near $96 \mathrm{~km}$ altitude. The absence of instabilities near $96 \mathrm{~km}$ is most probably due to the extremely low neutral winds at this altitude. Notice that neutral wind values are very small near $96 \mathrm{~km}$, but its magnitude rapidly increases below or above this altitude.

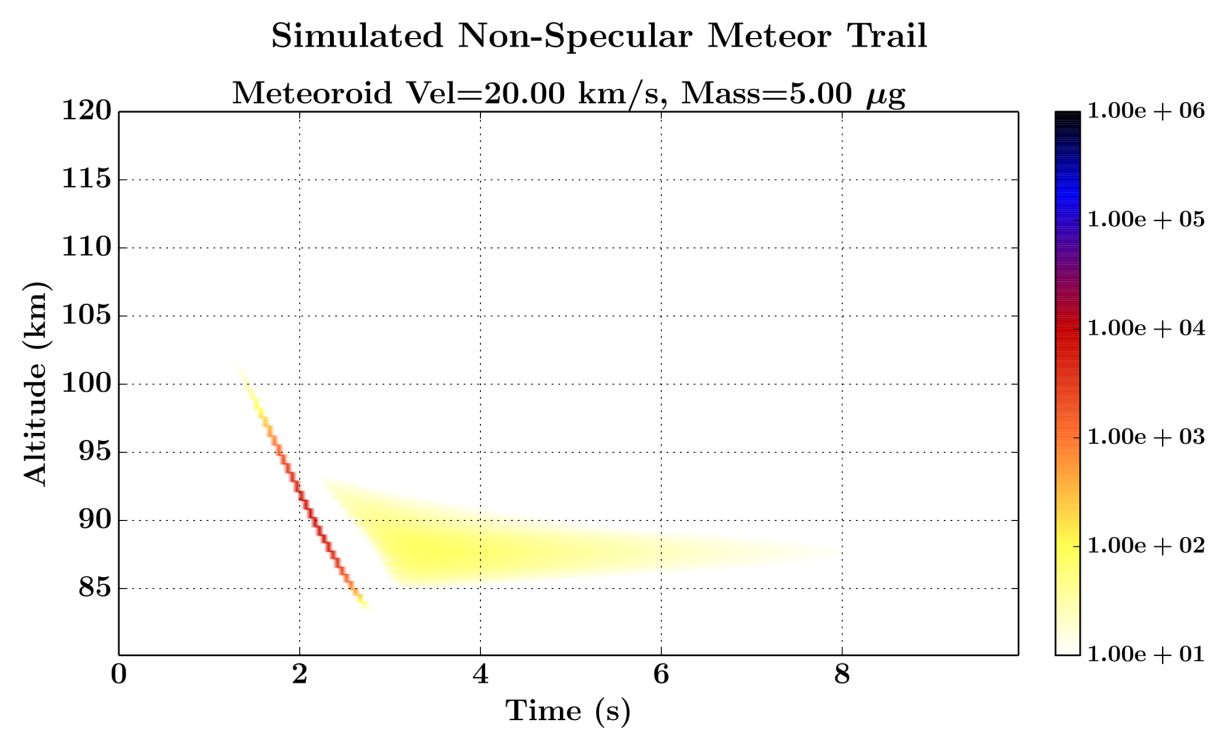

Figure 6. Meteor simulation to investigate the effect of weak neutral winds. In this example, instabilities take around $500 \mathrm{~ms}$ to develop due to neutral winds of $2.2 \mathrm{~m} \mathrm{~s}^{-1}$.

\subsection{Effects of neutral wind shears on the characteristics of non-specular echoes}

As discussed above, the meteor simulations presented in Fig. 5 indicate that plasma instabilities cannot be sustained in the trail when horizontal neutral winds are weaker than a critical value. The nature of this behavior is because electron drift plasma instabilities at these altitudes are mainly composed of diamagnetic drifts that are divergence-free and therefore do not contribute to driving plasma instabilities (Oppenheim et al., 2001, 2003; Dyrud et al., 2002). An ob- servational VHF backscatter power map showing a head echo and non-specular meteor echoes over Salinas, Puerto Rico, at 06:15 LT on 22 February 1998 is depicted in Fig. 7a. The main features of this experimental RTI are in good agreement with the simulation results presented in Fig. 5. A geometrical analysis between the backscatter power and the antenna beam width of the VHF radar demonstrates that the absence of backscattering echoes in the RTI is not due to the effect of the nulls of the antenna pattern. We also discarded fragmentation effects since there are no interference signatures visible at other ranges. Therefore, we think that wind shears 
are the most probable cause of the backscatter structures displayed in Fig. 7a.

Another VHF backscatter power map of head echo and non-specular echoes is shown in Fig. 7b. This RTI was collected at 01:22 LT on 23 February 1998 over Salinas, Puerto Rico. We conducted more than 1000 meteoroid simulations under different horizontal neutral wind profiles, meteoroid masses, and speed ranges that were mentioned in Sect. 3 and concluded that the meteor reflections shown in Fig. $7 \mathrm{~b}$ could be reproduced using neutral wind shear conditions with a valley that expands over a couple of kilometres in range, near $150 \mathrm{~km}$, and a meteoroid mass of about $0.32 \mu \mathrm{g}$, traveling at $30 \mathrm{~km} \mathrm{~s}^{-1}$. Similarly to Fig. 7a, an analysis of the backscatter power of both head echo and the trail from Fig. 7b revealed that the abrupt cessation of non-specular trails near the $150 \mathrm{~km}$ range cannot be reproduced with sharp change values in trail densities or antenna beam-width nulls. Thus, the wind-condition-described lines above represent a viable explanation.

We went a step further and expanded our meteor simulation results to analyze meteor trails collected with the highpower large-aperture Jicamarca VHF radar. Since this radar has more power than the radar used during the Coqui-II campaign, it can routinely detect many pairs of head echo and non-specular trail echoes. For example, on 6 May 2007, between 04:45 and 05:10 LT, the Jicamarca radar collected 17 (out of 103) pairs of head echo and non-specular trails that displayed an absence of backscatter echoes near $104.5 \mathrm{~km}$ altitude. Two of these types of events are shown in Fig. 8a and b. Routine meteor interferometry analysis, described in Chau and Galindo (2008), was applied to the events presented in Fig. $8 \mathrm{a}$ and b. We analyzed each of the head echo events using both received signal-to-noise ratio (SNR) and interferometry analysis. We discarded noise level as a potential explanation since, in the examples we report, all trail echoes were at least $3 \mathrm{~dB}$ above the noise level, so statistically, it is improbable that noise is responsible for gaps in different echoes in the same range and around the same time. We also discarded antenna nulls as a possible explanation for the trails' gaps since interferometry analysis placed these events in the main lobe of the antenna. These examples from Jicamarca have echo gaps observed only in the trail echoes, as shown in Fig. 8a. Notice that there is no drop in power intensity for the head echo around the $104.5 \mathrm{~km}$ range when zoomed in on this figure. The head echo event shown in Fig. 8a started at 04:46 LT and was moving at a speed of $65 \mathrm{~km} \mathrm{~s}^{-1}$ in a direction almost parallel to the geographic east-west direction. The event shown in Fig. 8b started at 04:54 LT and was moving with a speed of $56 \mathrm{~km} \mathrm{~s}^{-1}$ in the northwesterly direction. A full analysis of the 17 observational meteor events revealed meteoroids with different properties of mass, speed, or direction. Since neutral and electron densities typically do no exhibit abrupt changes in altitude, it is reasonable to conclude that neutral wind shears with very low amplitudes are the most probable cause of the absence of backscatter re- flections near $104.5 \mathrm{~km}$, as illustrated in these two events of Fig. $8 \mathrm{a}$ and b. Since the events shown in Fig. 8 last less than $3 \mathrm{~s}$ and therefore the method described in Oppenheim et al. (2009) to estimate neutral winds would not work, we expect to carry out both uncoded and coded radar experiments using the Jicamarca high-power large-aperture radar and an all-sky meteor radar to compute neutral wind amplitudes using meteor trails similar to the approach described in (Oppenheim et al., 2009, 2014) and Li et al. (2012) to establish a complete understanding and characterization of non-specular echoes.

Malhotra and Mathews (2009) reported meteor events mostly observed below $90 \mathrm{~km}$ altitude with the Jicamarca VHF radar, but some events were also detected above $110 \mathrm{~km}$. They called these events low-altitude trail echoes (LATE) because the majority of these events occurred below $90 \mathrm{~km}$ and did not show the time gap between the head echo and the non-specular trails needed for plasma instabilities to manifest and grow. They noted that meteoroids with high mass could penetrate these low altitudes and hypothesize that fragmentation and high neutral density at these low altitudes should be considered in the characterization of these LATE events. Sugar et al. (2010) suggested that pockets of material with lower sublimation temperatures in the meteoroid could be the cause of these LATE events. They explained that once these pockets of meteoroid materials were released, plasma irregularities could rapidly develop in the trail due to a higher density of plasma generated by these pockets. In contrast, we provide an alternative explanation based on the results reported in this paper. We suggest that it is possible for neutral winds to be a mechanism that can influence the generation of LATE echoes. For example, Fig. 9 shows a meteor simulation obtained using a $0.5 \mu \mathrm{g}$ meteoroid traveling at $34 \mathrm{~km} \mathrm{~s}^{-1}$. In this example, neutral wind shears affect the production of electrons in the final stage of the meteor trail, resulting in a trail echo spanning $\sim 1 \mathrm{~km}$ in range that has similar spacetime signatures to LATE echoes. Notice that the creation of LATE events based on our findings requires an unusual combination of meteoroid occurrence and atmosphere background conditions, making LATE events rare. However, our results are not limited to the final stages of the meteoroid occurrence. We also expect to see LATE-like events at the initial stage of the meteoroid passage if the right conditions, such as background electron density and winds, are satisfied. Nonetheless, low sublimation temperatures are also required to rapidly generate instabilities; the proper combination of meteoroid and atmosphere properties would also create similar echoes at initial stages of the meteor trail as presented in Sugar et al. (2010).

\subsection{Time delay between head echo and non-specular meteor trail}

Reported VHF radar meteor events typically show time delays between head echo and non-specular meteor trail that can vary from 0 to $\sim 1$ s (Mathews, 2004; Malhotra et al., 
(a)

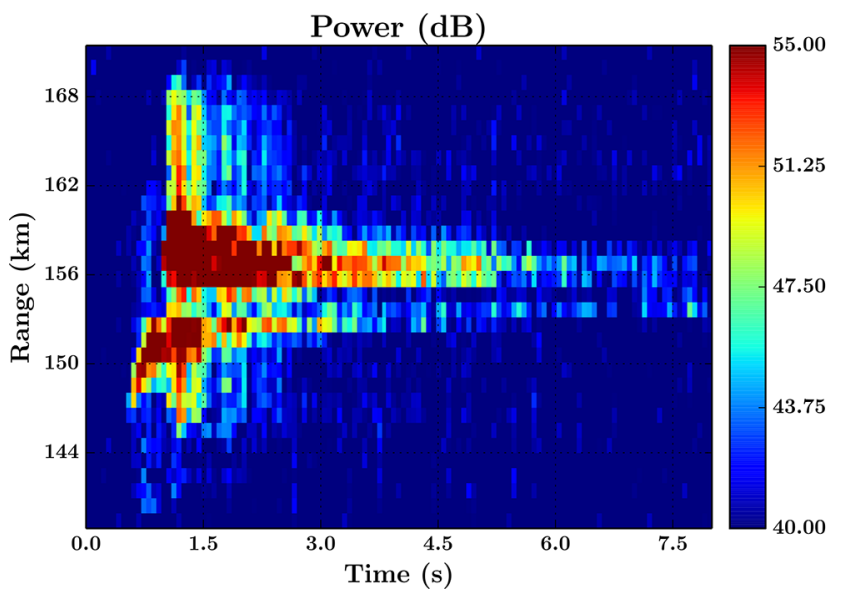

(b)

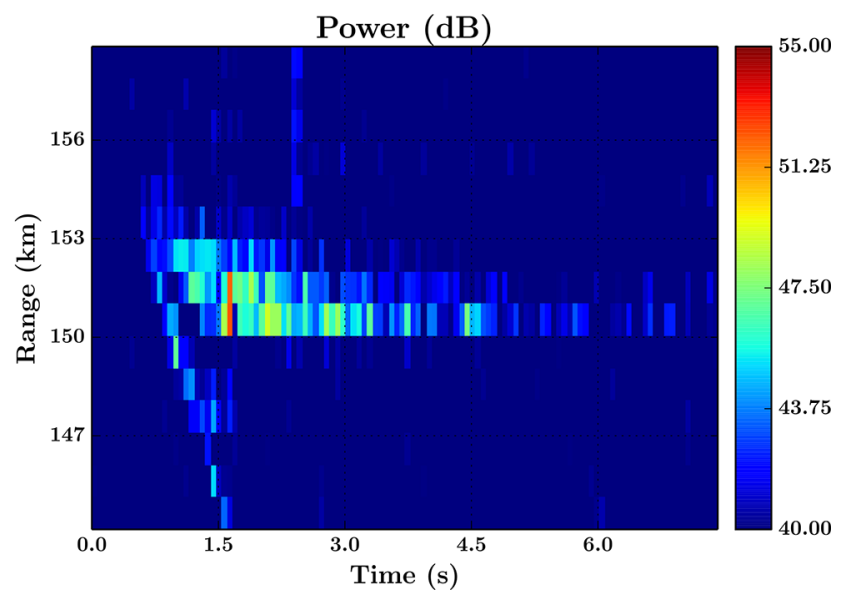

Figure 7. Meteor trails observed with the VHF radar operated in Salinas, Puerto Rico, during the Coqui-II campaign. Panel (a) exhibits absence of backscattering power between the 154 and $155 \mathrm{~km}$ range that resembles our simulation results shown in Fig. 5, while panel (b) shows that backscattering signals have a sudden decrease in ranges below $150 \mathrm{~km}$.

(a)

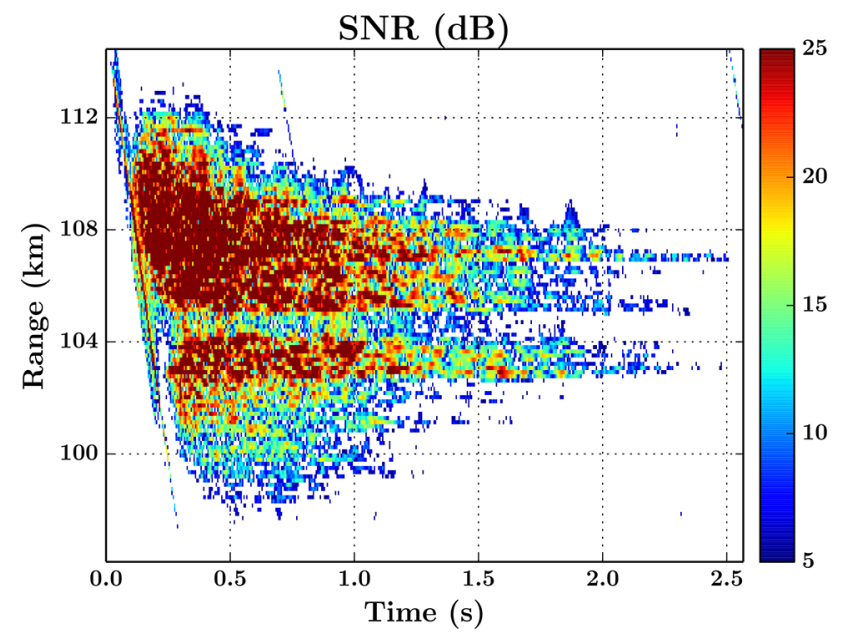

(b)

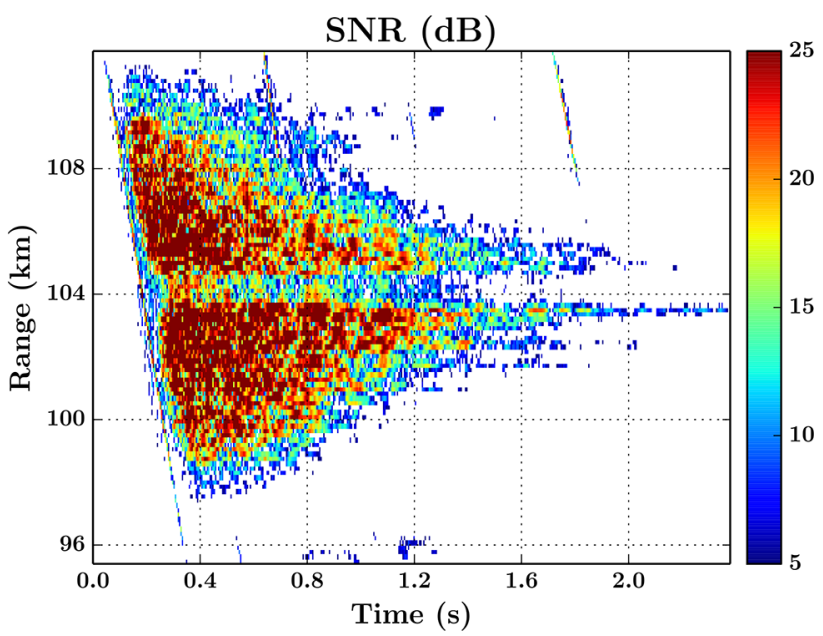

Figure 8. (a, b) show meteor trails collected with Jicamarca VHF radar at 04:46 and 04:54 LT, respectively, on 5 May 2007. The meteoroid in (a) travels at $65 \mathrm{~km} \mathrm{~s}^{-1}$, almost parallel to the east-west magnetic direction, while the meteoroid in (b) moves in the northwesterly magnetic direction at a speed of $56 \mathrm{~km} \mathrm{~s}^{-1}$. Notice that both examples exhibit a sudden decrease in the backscatter power near $104.5 \mathrm{~km}$ altitude.

2007). Time delays on the order of 20 to $30 \mathrm{~ms}$ have been associated with the timescales needed to generate plasma turbulence in the trail (Dyrud et al., 2002), while much longer time-delay values have been attributed to the time that is required to transport meteor plasma to regions where instabilities develop (Mathews, 2004). Also, Malhotra et al. (2007) have shown that the time delay can also be a function of the viewing geometry between the radar $\boldsymbol{k}$ vector and the Earth's magnetic field $\boldsymbol{B}$. However, our meteor simulations reported in this paper present an additional scenario. For example, Fig. 6 demonstrates that time delays on the order of hundreds of milliseconds or longer can be generated when me- teoroids have masses greater than $5 \mu \mathrm{g}$ and produce a dense non-specular trail that is affected by neutral winds with amplitudes close to the critical value, which are required to sustain plasma instabilities. Our simulation results imply that instabilities slowly develop in these meteor trails because both polarization drifts and neutral winds do not have the strength to drive instabilities in the meteor trail rapidly. We should notice that diamagnetic drifts are divergence-free and therefore do not drive instabilities (Oppenheim et al., 2003). 


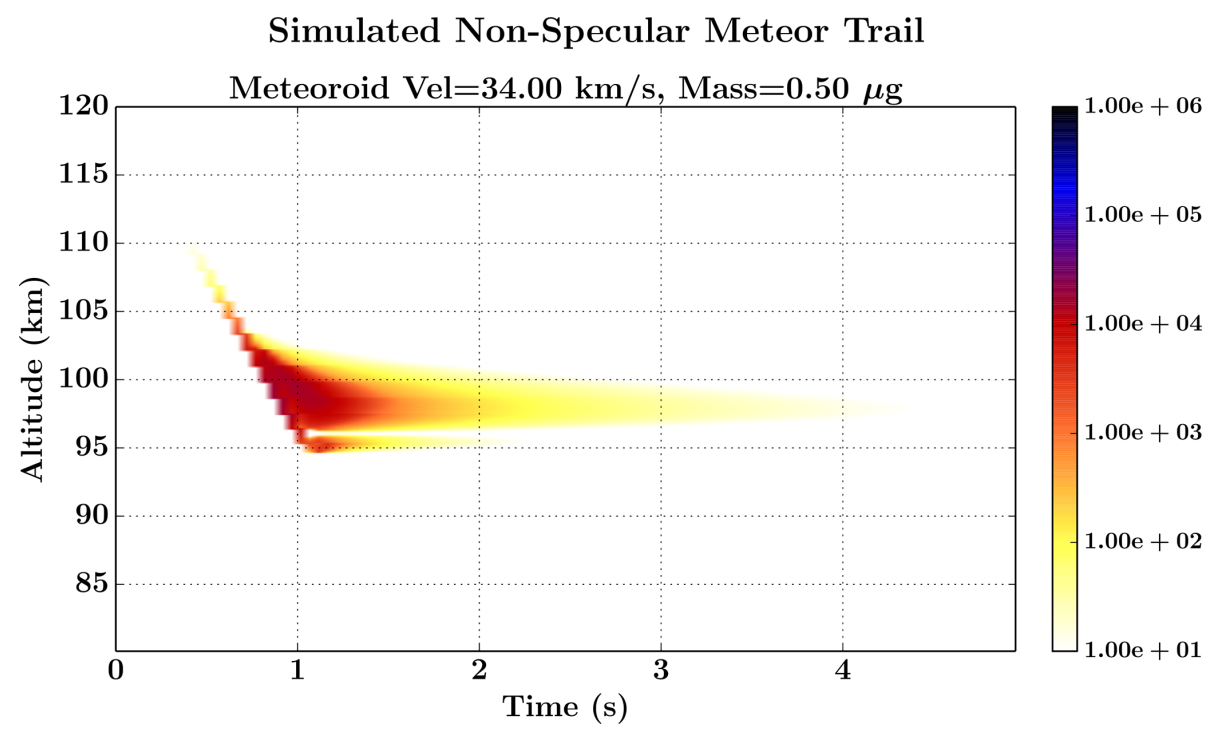

Figure 9. Simulation showing the effects of neutral wind shears, using a meteoroid with a mass of $0.5 \mu \mathrm{g}$ that is traveling at $34 \mathrm{~km} \mathrm{~s}^{-1}$. The background atmosphere/ionosphere conditions have a vertical profile similar to those displayed in Fig. 5.

\section{Conclusions}

This paper presented computer simulations of meteor trails over Salinas, Puerto Rico, to understand the effects of neutral winds on meteor trail evolution. The non-specular meteor trail echo simulator (NSMES), which we described in this paper, is an important tool to study non-specular meteor trail echoes under different meteoroid masses and atmospheric conditions. It can be used to compare simulation results with experimental observations. For example, we showed using NSMES that it is plausible for meteor trails to exhibit unique radar signatures due to neutral winds that are useful for creating a comprehensive characterization of meteor trails. We also discussed how time delays between head echo and non-specular trail on the order of hundreds of milliseconds or longer could be the result of dense trails that are affected by weak neutral wind amplitudes. Also, we provided experimental evidence that supported our simulation results and discussed their implications for other locations such as the Jicamarca VHF radar. It is not the purpose of this short paper to analyze all competing ideas in head echo and nonspecular meteor trail research but to provide an alternative explanation of this research topic. We plan to fine-tune our meteor model and make the code open source to the broader scientific community so others can verify our findings or expand our studies. We envision these efforts as not replacing but rather complementing more complex 3D meteor models (Oppenheim and Dimant, 2015; Dimant and Oppenheim, 2017a, b).

Code and data availability. The data set for this paper is available online (http://www.datacommons.psu.edu/, DOI: https://doi. org/10.26208/38vd-ne79, Galindo, 2021); following the data policy, for details of this data and code, please contact Julio Urbina at jvu1@psu.edu.

Author contributions. FG, JU, and LD conceived the project. FG did a significant part of the simulation and data analysis work. JU and LD helped with the discussion and interpretation of the results.

Competing interests. The authors declare that they have no conflict of interest.

Disclaimer. Publisher's note: Copernicus Publications remains neutral with regard to jurisdictional claims in published maps and institutional affiliations.

Acknowledgements. The authors would like to thank the JRO (and IGP) staff for performing the observations and Robert Sorbello for helping us in the preparation of the manuscript. The Jicamarca Radio Observatory is a facility of the Instituto Geofisico del Peru operated with support from NSF AGS0905448 through Cornell University.

Financial support. This research has been supported by the National Science Foundation (grant nos. AGS0638624 and AGS0457156).

Review statement. This paper was edited by Keisuke Hosokawa and reviewed by two anonymous referees. 


\section{References}

Bilitza, D.: IRI the International Standard for the Ionosphere, Adv. Radio Sci., 16, 1-11, https://doi.org/10.5194/ars-16-12018, 2018.

Bilitza, D. and Reinisch, B.: International Reference Ionosphere 2007: Improvements and new parameterse, Adv. Space Res., 85, 599-609, 2008.

Ceplecha, Z., Borovička, J., Elford, W., ReVelle, D., Hawkes, R., Porubčan, V., and Šimek, M.: Meteor Phenomena and Bodies, Space Sci. Rev., 84, 327-471, https://doi.org/10.1023/A:1005069928850, 1998.

Chapin, E. and Kudeki, E.: Plasma wave excitation on meteor trails in the equatorial electrojet, Geophys. Res. Lett., 21, 2433-2436, https://doi.org/10.1029/94GL01705, 1994.

Chau, J. L. and Galindo, F.: First definitive observations of meteor shower particles using a high-power large-aperture radar, Icarus, 194, 23-29, https://doi.org/10.1016/j.icarus.2007.09.021, 2008.

Chau, J. L., Strelnikova, I., Schult, C., Oppenheim, M. M., Kelley, M. C., Stober, G., and Singer, W.: Nonspecular meteor trails from non-field-aligned irregularities: Can they be explained by presence of charged meteor dust?, Geophys. Res. Lett., 41, 33363343, https://doi.org/10.1002/2014GL059922, 2014.

Close, S., Oppenheim, M., Hunt, S., and Coster, A.: A technique for calculating meteor plasma density and meteoroid mass from radar head echo scattering, Icarus, 168, 43-52, https://doi.org/10.1016/j.icarus.2003.11.018, 2004.

Dimant, Y. S. and Oppenheim, M. M.: Formation of plasma around a small meteoroid: 1. Kinetic theory, J. Geophys. Res.-Space, 122, 4669-4696, https://doi.org/10.1002/2017JA023960, 2017a.

Dimant, Y. S. and Oppenheim, M. M.: Formation of plasma around a small meteoroid: 2. Implications for radar head echo, J. Geophys. Res.-Space, 122, 4697-4711, https://doi.org/10.1002/2017JA023963, 2017b.

Drob, D. P., Emmert, J. T., Crowley, G., Picone, J. M., Shepherd, G. G., Skinner, W., Hays, P., Niciejewski, R. J., Larsen, M., She, C. Y., Meriwether, J. W., Hernandez, G., Jarvis, M. J., Sipler, D. P., Tepley, C. A., O’Brien, M. S., Bowman, J. R., Wu, Q., Murayama, Y., Kawamura, S., Reid, I. M., and Vincent, R. A.: An empirical model of the Earth's horizontal wind fields: HWM07, J. Geophys. Res.-Space, 113, A12304, https://doi.org/10.1029/2008JA013668, 2008.

Drob, D. P., Emmert, J. T., Meriwether, J. W., Makela, J. J., Doornbos, E., Conde, M., Hernandez, G., Noto, J., Zawdie, K. A., McDonald, S. E., Huba, J. D., and Klenzing, J. H.: An update to the Horizontal Wind Model (HWM) : The quiet time thermosphere, Earth and Space Science, 2, 301-319, https://doi.org/10.1002/2014EA000089, 2015.

Dyrud, L. and Janches, D.: Modeling the meteor head echo using Arecibo radar observations, J. Atmos. Sol.-Terr. Phy., 70, 16211632, https://doi.org/10.1016/j.jastp.2008.06.016, 2008.

Dyrud, L. P., Oppenheim, M. M., and vom Endt, A. F.: The anomalous diffusion of meteor trails, Geophys. Res. Lett., 28, 27752778, https://doi.org/10.1029/2000GL012749, 2001.

Dyrud, L. P., Oppenheim, M. M., Close, S., and Hunt, S.: Interpretation of non-specular radar meteor trails, Geophys. Res. Lett., 29, 2012, https://doi.org/10.1029/2002GL015953, 2002.

Dyrud, L. P., Ray, L., Oppenheim, M., Close, S., and Denney, K.: Modelling high-power large-aperture radar me- teor trails, J. Atmos. Sol.-Terr. Phy., 67, 1171-1177, https://doi.org/10.1016/j.jastp.2005.06.016, 2005.

Dyrud, L. P., Kudeki, E., and Oppenheim, M.: Modeling long duration meteor trails, J. Geophys. Res.-Space, 112, a12307, https://doi.org/10.1029/2007JA012692, 2007.

Dyrud, L. P., Urbina, J., Fentzke, J. T., Hibbit, E., and Hinrichs, J.: Global variation of meteor trail plasma turbulence, Ann. Geophys., 29, 2277-2286, https://doi.org/10.5194/angeo-29-22772011, 2011.

Galindo, F., Urbina, J., and Dyrud, L.: Effect of neutral winds on the creation of non-specular meteor trail echoes, The Pennsylvania State University [data set], https://doi.org/10.26208/38vd-ne79, 2021.

Hinrichs, J., Dyrud, L. P., and Urbina, J.: Diurnal variation of non-specular meteor trails, Ann. Geophys., 27, 1961-1967, https://doi.org/10.5194/angeo-27-1961-2009, 2009.

Li, G., Ning, B., Hu, L., Chu, Y.-H., Reid, I. M., and Dolman, B. K.: A comparison of lower thermospheric winds derived from range spread and specular meteor trail echoes, J. Geophys. Res.-Space, 117, A03310, https://doi.org/10.1029/2011JA016847, 2012.

Malhotra, A. and Mathews, J. D.: Low-altitude meteor trail echoes, Geophys. Res. Lett., 36, 121106, https://doi.org/10.1029/2009GL040558, 2009.

Malhotra, A., Mathews, J. D., and Urbina, J.: A radio science perspective on long-duration meteor trails, J. Geophys. Res.-Space, 112, A12303, https://doi.org/10.1029/2007JA012576, 2007.

Mathews, J.: Radio science issues surrounding HF/VHF/UHF radar meteor studies, J. Atmos. Sol.-Terr. Phy., 66, 285-299, https://doi.org/10.1016/j.jastp.2003.11.001, 2004.

Mathews, J. D., Janches, D., Meisel, D. D., and Zhou, Q. H.: The micrometeoroid mass flux into the upper atmosphere: Arecibo results and a comparison with prior estimates, Geophys. Res. Lett., 28, 1929-1932, https://doi.org/10.1029/2000GL012621, 2001.

Oppenheim, M. M. and Dimant, Y. S.: First 3-D simulations of meteor plasma dynamics and turbulence, Geophys. Res. Lett., 42, 681-687, https://doi.org/10.1002/2014GL062411, 2015.

Oppenheim, M. M., Dyrud, L. P., Close, S., and Hunt, S.: Theory and simulations of field-aligned irregularities in meteor trails, in: Meteoroids 2001 Conference, edited by: Warmbein, B., ESA Sp. Publ., 495, 361-366, 2001.

Oppenheim, M. M., Dyrud, L. P., and Ray, L.: Plasma instabilities in meteor trails: Linear theory, J. Geophys. Res.-Space, 108, 1063, https://doi.org/10.1029/2002JA009548, 2003.

Oppenheim, M. M., Sugar, G., Slowey, N. O., Bass, E., Chau, J. L., and Close, S.: Remote sensing lower thermosphere wind profiles using non-specular meteor echoes, Geophys. Res. Lett., 36, L09817, https://doi.org/10.1029/2009GL037353, 2009.

Oppenheim, M. M., Arredondo, S., and Sugar, G.: Intense winds and shears in the equatorial lower thermosphere measured by high-resolution nonspecular meteor radar, J. Geophys. Res.Space, 119, 2178-2186, https://doi.org/10.1002/2013JA019272, 2014.

Picone, J. M., Hedin, A. E., Drob, D. P., and Aikin, A. C.: NRLMSISE-00 empirical model of the atmosphere: Statistical comparisons and scientific issues, J. Geophys. Res.-Space, 107, 1468, SIA 15-1-SIA 15-16, https://doi.org/10.1029/2002JA009430, 2002.

Sugar, G., Oppenheim, M. M., Bass, E., and Chau, J. L.: Nonspecular meteor trail altitude distributions and durations observed 
by a $50 \mathrm{MHz}$ high-power radar, J. Geophys. Res.-Space, 115, A12334, https://doi.org/10.1029/2010JA015705, 2010.

Sugar, G., Oppenheim, M. M., Dimant, Y. S., and Close, S.: Formation of Plasma Around a Small Meteoroid: Simulation and Theory, J. Geophys. Res.-Space, 123, 4080-4093, https://doi.org/10.1002/2018JA025265, 2018.

Sugar, G., Oppenheim, M. M., Dimant, Y. S., and Close, S.: Formation of Plasma Around a Small Meteoroid: Electrostatic Simulations, J. Geophys. Res.-Space, 124, 3810-3826, https://doi.org/10.1029/2018JA026434, 2019.

Urbina, J., Kudeki, E., Franke, S. J., Gonzalez, S., Zhou, Q., and Collins, S. C.: $50 \mathrm{MHz}$ radar observations of mid-latitude E-region irregularities at Camp Santiago, Puerto Rico, Geophys. Res. Lett., 27, 2853-2856, https://doi.org/10.1029/2000GL000028, 2000.
Yee, J. and Close, S.: Plasma turbulence of nonspecular trail plasmas as measured by a high-power large-aperture radar, J. Geophys. Res.-Atmos., 118, 13449-13462, https://doi.org/10.1002/2013JD020247, 2013.

Zhou, Q. H., Mathews, J. D., and Nakamura, T.: Implications of meteor observations by the MU Radar, Geophys. Res. Lett., 28, 1399-1402, https://doi.org/10.1029/2000GL012504, 2001. 